\title{
Effects of a 24-h naproxen dose on hydration and electrolyte measures during moderate-intensity cycling in the heat
}

\author{
Dawn M. Emerson $^{a \star \dagger}$, Toni M. Torres-McGehee ${ }^{\mathrm{a}}$, J. Mark Davis ${ }^{\mathrm{a}}$, Stephen C.L. Chen ${ }^{\mathrm{a} \ddagger}$, J. Larry \\ Durstine $^{a}$, Craig E. Pfeifer ${ }^{\mathrm{a}}$, Charles C. Emerson ${ }^{\mathrm{a} \|}$, Justin V. Stone ${ }^{\mathrm{a}}$, and Joseph D. Bivona ${ }^{\mathrm{a}}$ \\ ${ }^{a}$ Department of Exercise Science, Public Health Research Center, University of South Carolina, \\ 921 Assembly Street, Columbia, SC 29208, USA \\ *dawn.emerson@ku.edu \\ ${ }^{\dagger}$ Present address: University of Kansas, Lawrence, KS 66045, USA \\ ${ }^{\ddagger}$ Present address: Bob Jones University, Greenville, SC 29614, USA \\ ${ }^{\S}$ Present address: Lander University, Greenwood, SC 29649, USA \\ "Present address: University of Missouri-Kansas City, Kansas City, MO 64110, USA
}

\section{OPEN ACCESS}

\section{Citation: Emerson DM, Torres- \\ McGehee TM, Davis JM, Chen SCL, Durstine JL, Pfeifer CE, Emerson CC, Stone JV, and Bivona JD. 2017. Effects of a 24-h naproxen dose on hydration and electrolyte measures during moderate- intensity cycling in the heat. FACETS 2: 819-832. doi: I 0.1 I 39/facets-20 17-0042 \\ Editor: Angelo Belcastro \\ Received: April 20, 2017}

Accepted: August 7, 2017

Published: October 24, 2017

Copyright: (C) 2017 Emerson et al. This work is licensed under a Creative Commons Attribution 4.0 International License (CC BY 4.0), which permits unrestricted use, distribution, and reproduction in any medium, provided the original author(s) and source are credited.

Published by: Canadian Science Publishing

\begin{abstract}
Few controlled laboratory studies have examined the negative effects non-steroidal anti-inflammatory drugs can have on fluid-electrolyte balance during exercise. Our objective was to determine whether a 24-h naproxen dose negatively affected hydration and electrolyte measures before, during, and $3 \mathrm{~h}$ after 90 min of cycling in a hot or ambient environment. Using a double blind, randomized and counterbalanced cross-over design, 11 volunteers (six male, five female) completed four trials, with conditions as follows: (1) placebo and ambient, (2) placebo and heat, (3) naproxen and ambient, and (4) naproxen and heat. We found no statistically significant differences among experimental conditions for any dependent measures. Though not statistically significant, mean fluid volume was higher and urine volume was lower during naproxen trials compared with placebos. Mean aggregate plasma sodium was $<135 \mathrm{mmol} / \mathrm{L}$ at all time points and did not significantly change over time. Overall plasma potassium significantly increased pre- $(3.9 \pm 0.4)$ to post-exercise $(4.2 \pm 0.4 \mathrm{mmol} / \mathrm{L}, p=0.02)$. In conclusion, an acute naproxen dose did not significantly alter hydration-electrolyte balance. The trend for naproxen to increase fluid volume and decrease urine volume suggests the start of fluid retention, which should concern individuals at risk for hyponatremia or with pre-existing cardiovascular conditions.
\end{abstract}

Key words: NSAID, fluid volume, urine volume, plasma sodium, plasma potassium

\section{Introduction}

Non-steroidal anti-inflammatory drugs (NSAIDs) include a variety of prescription and non-prescription medications including aspirin, ibuprofen, and naproxen. An individual drug's effectiveness and side effects are dependent on the medication's chemical makeup and selectivity toward inhibiting cyclooxygenase (COX). Two COX derivatives $(-1$ and -2$)$ are found in humans. Inherently located in most tissues, COX-1 is responsible for regulating gastric mucosa secretions, platelet aggregation (clotting), and renal blood flow (Constanti et al. 1998). COX-2, present in very small amounts naturally, is upregulated locally by inflammatory mediators (e.g., cytokines) and facilitates inflammation, 
pain, and fever (Constanti et al. 1998). Anti-inflammatory and analgesic NSAID effects occur by preventing COX from catalyzing the conversion of arachidonic acid (fatty acid released from cell membranes during injury/inflammation) to prostaglandin (PG). Numerous PG subsets are responsible for not only promoting pain and inflammation, but also maintaining blood pressure (BP), plasma volume, and renal function. Less imperative in normal healthy individuals, PGs are critical in maintaining homeostasis during altered cardiovascular states (e.g., hypotension) or altered fluid regulation (e.g., hypovolemia and sodium imbalance) (Garella and Matarese 1984)-common conditions experienced during physical activity. Inhibiting PGs can perpetuate altered cardiovascular and renal function, potentially leading to serious fluid and electrolyte imbalances (e.g., hyponatremia and hypertension) or even acute renal failure (Garella and Matarese 1984; Rosner and Kirven 2007).

Existing literature on NSAIDs and fluid regulation during physical activity primarily includes observational and retrospective cases of exertional hyponatremia (plasma sodium concentration $\left(\mathrm{P}\left[\mathrm{Na}^{+}\right]\right)$ $<135 \mathrm{mmol} / \mathrm{L}$ ) (Almond et al. 2005; Wharam et al. 2006; Rosner and Kirven 2007). NSAIDs are thought to potentiate hyponatremia by decreasing urine production and increasing water retention, which dilutes $\mathrm{P}\left[\mathrm{Na}^{+}\right]$(Cheng and Harris 2005). To our knowledge, there is one controlled study examining this relationship, which found that a $600 \mathrm{mg}$ dose of ibuprofen before and a $1200 \mathrm{mg}$ dose during a $160-\mathrm{km}$ race (with a temperature range $9-30^{\circ} \mathrm{C}$ ) did not significantly alter plasma electrolytes (Dumke et al. 2007). Controlled laboratory studies examining NSAID effects on fluid-electrolyte balance during exercise are lacking. Thus, as part of a larger study, we sought to determine acute dose effects of a commonly used over-the-counter NSAID, naproxen, on fluid and electrolyte measures during moderateintensity endurance exercise in a hot or ambient environment. We hypothesized naproxen would increase hydration (i.e., euhydration or hyperhydration) compared with placebo controls. We also hypothesized naproxen would significantly decrease $\mathrm{P}\left[\mathrm{Na}^{+}\right]$compared with placebo controls.

\section{Methods}

\section{Participants}

Seventeen participants (12 male, five female) began the study. Two participants withdrew from the study due to the exercise intensity and pills, three due to the time commitment, and one due to a non-study-related injury. Our final sample size was 11 (six male, five female). Prior to participation, participants read and signed an informed consent form approved by the University of South Carolina Institutional Review Board.

Potential participants were recruited from the University of South Carolina and the local Columbia, South Carolina community via posted flyers and word of mouth. To be considered for the study, participants completed a health and injury history questionnaire and were accepted if they were free of cardiovascular, respiratory, metabolic, musculoskeletal, fluid-electrolyte, gastrointestinal, and swallowing disorders. Potential participants then completed a graded cycling $\mathrm{V}_{2 \max }$ test to determine qualification as moderately trained $\left(\mathrm{V}_{2} \max 32-40 \mathrm{~mL} /(\mathrm{kg} \cdot \mathrm{min})\right)$ and familiarize participants with the stationary cycle. The health and injury questionnaire also included information on current prescription and non-prescription medication use, and females answered questions about their menstrual cycle and birth control. Baseline anthropometric measures included height (self-reported), weight, and body fat percentage (measured using a Tanita SC-331S Body Composition Monitor, Tanita Co., Tokyo, Japan).

\section{Experimental design and procedures}

A schematic of the experimental procedures is shown in Figure 1. Using a double-blind, randomized and counterbalanced, cross-over design, we evaluated the effect of naproxen or placebo in a hot or ambient environment. Participants completed trials under the following four experimental conditions: 


Information session

- Informed consent

- Health information

- Cycling $\dot{V}_{2 \text { max }}$ test

- Experimental

condition

assignments

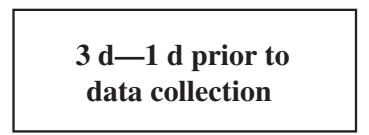

$-3 d$

- Begin diet and

physical activity log

- No vigorous

exercise

$-1 \mathrm{~d}$

- Written and verbal take home instructions

- 2 pills

\begin{tabular}{|c|c|c|c|}
\hline \multicolumn{4}{|c|}{ Data collection (4 trials) } \\
\hline Arrive to lab & 90 min cycling & Post-exercise & $\begin{array}{l}3 \text { h post- } \\
\text { exercise }\end{array}$ \\
\hline $\begin{array}{l}\text { - Confirm } 2 \text { pills } \\
\text { taken } \\
\text { - Give 3rd pill } \\
\text { - Baseline } \\
\text { - Blood } \\
\text { - Urine }\end{array}$ & $\begin{array}{l}\text { - Continuous HR } \\
\text { - } 3.5 \mathrm{~mL} / \mathrm{kg} \\
\text { water every } 15 \\
\text { min }\end{array}$ & $\begin{array}{l}\text { - Blood } \\
\text { - Urine } \\
\text { - BM } \\
\text { - BP and HR }\end{array}$ & $\begin{array}{l}\text { - Water ad libitum } \\
\text { - Post } \\
\text { - Blood } \\
\text { - Urine } \\
\text { - BM } \\
\text { - BP and HR }\end{array}$ \\
\hline
\end{tabular}

- HR and BP

Fig. 1. Schematic of the experimental procedures. Blood measures include plasma osmolality, plasma sodium concentration, and plasma potassium concentration. Urine measures include urine osmolality and urine specific gravity. BM, body mass; BP, blood pressure; HR, heart rate.

(1) placebo and ambient (Control), (2) placebo and heat (Heat), (3) naproxen and ambient (Npx), and (4) naproxen and heat (NpxHeat). A minimum of $7 \mathrm{~d}$ separated each trial. A 24-h dose (three capsules) of placebo (cellulose) or naproxen (220 mg naproxen sodium per dose) was given to participants with instructions to take one capsule at 16,8 , and $0 \mathrm{~h}$ prior to data collection. Instructions to take each pill (i.e., timing, with at least $8 \mathrm{oz}$ of fluid and not with food) were based on Aleve ${ }^{\circledR}$ manufacturer directions. To blind participants and primary investigators to naproxen or placebo trials, a local pharmacy compounded pills to appear the same. A research assistant prepared coded envelopes containing pills for each trial and randomly assigned participants to an experimental order prior to data collection.

To control for the effects of dietary and physical activity pre-data collection, participants tracked diet and physical activity for $3 \mathrm{~d}$ prior and $1 \mathrm{~d}$ after data collection using the online nutrition software FoodProdigy $^{\mathrm{TM}}$ (ESHA Research, Salem, Oregon, USA). Participants were instructed to refrain from intense, vigorous exercise $48 \mathrm{~h}$ prior to data collection (Peters et al. 2000). Twenty-four hours prior, participants were provided written and verbal instructions for taking two pills (placebo or naproxen), to consume a small meal prior to reporting to the laboratory, consume fluids to promote euhydration, refrain from all physical activity, and attempt to sleep a "normal" amount. Using the diet and physical activity habits from the $24 \mathrm{~h}$ prior to the first trial, participants were instructed to mimic the same diet and physical activity habits $24 \mathrm{~h}$ prior to each subsequent trial. Participants were also instructed to not take any other analgesic or anti-inflammatory medications during the course of the study.

Upon arrival to the laboratory for data collection and before taking the third pill, participants were verbally asked if they took their previous two pills. Baseline hydration, electrolyte, and cardiovascular measures were assessed prior to participants cycling for $90 \mathrm{~min}$ in either a hot (temperature $=35.7 \pm 1.3{ }^{\circ} \mathrm{C}$, relative humidity $(\mathrm{RH})=53.2 \% \pm 3.2 \%$ ) or ambient (temperature $=22.7 \pm 1.8^{\circ} \mathrm{C}, \mathrm{RH}=52.4 \% \pm 5.5 \%$ ) environment. Immediately following exercise, participants were weighed, provided a urine sample, and had blood collected. Participants then rested for $3 \mathrm{~h}$ in a semi-reclined/seated position in an ambient environment $\left(23^{\circ} \mathrm{C}, 56 \% \mathrm{RH}\right)$ where they were allowed to consume water ad libitum and given a snack. Due to the larger study, snacks were sugar free and quantity for each trial was based on baseline body mass (BM). Three hours after exercise, hydration, electrolyte, and cardiovascular measures were again assessed.

\section{Cycling protocol}

The 90-min cycling protocol was completed on a stationary bike (Monark Ergomedic 828E, Monark Exercise AB, Vansbro, Sweden). Prior to a 3-min warm up, participants were provided their target 
heart rate (HR), equivalent to $70 \% \dot{\mathrm{VO}}_{2 \max }$, to maintain during 80 min of steady-state cycling. Participants were instructed to reach the HR by the end of the 3-min warm up. At the end of $80 \mathrm{~min}$, participants performed $10 \mathrm{~min}$ at maximum effort, with each participant given verbal encouragement by research assistants. The protocol concluded with a 5-min cool down.

\section{Hydration and electrolyte measures}

Hydration measures included plasma osmolality (Posm), urine osmolality (Uosm), urine specific gravity (Usg), and percent change in body mass (\%BM). Participants were required to be euhydrated prior to beginning data collection (Posm $\leq 290 \mathrm{mOsm} / \mathrm{L}$, Uosm $<700 \mathrm{mOsm} / \mathrm{L}$, Usg $\leq 1.020$, and (or) $\% \mathrm{BM}<1 \%$ ) (Sawka et al. 2007). Immediate hydration status was assessed using Usg. Further hydration analysis was conducted using Posm, Uosm, and \%BM.

A research assistant collected blood from an antecubital vein into $6 \mathrm{~mL}$ lithium heparin vacutainer tubes pre-, immediately post-, and $3 \mathrm{~h}$ post-exercise. Tubes were inverted several times to mix and immediately placed on ice. Samples were centrifuged at $3000 \mathrm{rpm}$ for $15 \mathrm{~min}$. Plasma was pipetted into microtubes and stored at $-20^{\circ} \mathrm{C}$ until analysis. Osmolality was measured using freeze point depression (using a Multi-sample Osmometer model 2020, Advanced Instruments, Norwood, Massachusetts, USA). To determine changes in $\mathrm{P}\left[\mathrm{Na}^{+}\right]$and plasma potassium concentration $\left(\mathrm{P}\left[\mathrm{K}^{+}\right]\right.$), we used ion-selective electrodes (using an EasyLyte ${ }^{\circledR} \mathrm{Na} / \mathrm{K}$ electrolyte analyzer model REF 2277, Medica, Bedford, Massachusetts, USA). Normal $\mathrm{P}\left[\mathrm{Na}^{+}\right]$was defined as $>135 \mathrm{mmol} / \mathrm{L}$ (Rosner and Kirven 2007) and normal $\mathrm{P}\left[\mathrm{K}^{+}\right]<5 \mathrm{mmol} / \mathrm{L}$ (Clausen 2010).

Urine was obtained pre-, immediately post-, and $3 \mathrm{~h}$ post-exercise. Participants urinated a small amount before placing the specimen cup in midstream to collect a minimum of $1 \mathrm{oz}$ of urine. A handheld clinical refractometer (model REF 312, Atago Company Ltd., Tokyo, Japan) was used to measure Usg. Urine aliquots were stored in microtubes at $-20{ }^{\circ} \mathrm{C}$ until osmolality analysis. All urine produced after pre-hydration measures were collected into Uvol containers and urine cups (for Usg and Uosm measures). Total urine volume (Uvol) produced over the trial was measured using a graduated cylinder.

Pre-, immediately post-, and $3 \mathrm{~h}$ post-exercise BM was measured using a body composition analyzer (Tanita SC-331S Body Composition Monitor, Tanita Co., Tokyo, Japan). Participants dressed in shorts and a t-shirt and voided urine before stepping on the scale. Immediately post-exercise participants toweled off sweat before weighing. Participants were then allowed to change clothes and instructed to return with their "wet" clothes to be weighed. Differences in "wet" versus "dry" clothes' weight (measured at another time) were used to adjust BM for sweat loss. We calculated sweat rate using the formula: (pre-activity BM - post-activity BM + fluid volume consumed - urine volume)/ exercise time (Casa et al. 2000).

\section{Fluid volume measures}

To maintain hydration during exercise, participants were instructed to drink $3.5 \mathrm{~mL} / \mathrm{kg}$ of water every $15 \mathrm{~min}$. Participants were allowed to consume more than required if they desired. At the end of exercise, researchers measured total fluid volume (Fvol) consumed. During the 3-h rest period, participants consumed water ad libitum and researchers measured Fvol consumed during rest.

\section{Cardiovascular measures}

To ensure that participants maintained the target $\mathrm{HR}$ and remained at safe limits during exercise, HR was continuously monitored using Polar HR monitors (Polar Electro Inc., Lake Success, New York, USA). BP was measured using a standard sphygmomanometer and stethoscope. Measures were taken 
by a research assistant and recorded every 15 min during exercise. Pre-, immediately post-, and $3 \mathrm{~h}$ post-exercise HR and BP were assessed.

\section{Statistical analysis}

IBM SPSS Statistics (version XXII; IBM Corporation, Armonk, New York, USA) was used for all analyses. Descriptive statistics (mean and standard deviations) for all dependent variables were calculated. A one-way ANOVA was used to determine demographic differences between genders (e.g., age, height, weight). A 3 (time) $\times 4$ (condition) repeated measures ANOVA determined differences for plasma, urine, \%BM, HR, and BP. Greenhouse-Geisser corrections were used for $\mathrm{P}\left[\mathrm{Na}^{+}\right], \mathrm{BP}$, and HR because these variables violated sphericity. Post hoc analysis was conducted for significant main effects using Bonferroni corrections. We used a one-way ANOVA to determine differences among conditions and between genders for Fvol consumed during exercise, total Fvol, and Uvol. To control for BM, Fvol was corrected to $\mathrm{mL} / \mathrm{kg}$ and a one-way ANOVA was used to determined differences among conditions and between genders. Paired sample $t$ tests determined differences in sweat rate and Fvol during exercise. Using $\mathrm{G}^{\star}$ Power (version 3.1.9.2, Heinrich Heine University, Dusseldorf, Germany) (Faul et al. 2007), post hoc power calculation with means and variances for Usg and Uosm indicated that statistical power was 0.9 . Significance level was set at $p<0.05$ for all analyses.

\section{Results}

No significant differences were found between genders for age (27.8 \pm 5.7 years), weight $(79.1 \pm 17.9 \mathrm{~kg})$, $\dot{\mathrm{VO}}_{2 \max }(41.4 \pm 5.7 \mathrm{~mL} / \mathrm{kg} / \mathrm{min})$, sweat rate $(0.9 \pm 0.3 \mathrm{~L} / \mathrm{h})$, baseline resting HR $(52.6 \pm 6.6 \mathrm{bpm})$, baseline resting systolic $(115.6 \pm 18.7 \mathrm{~mm} \mathrm{Hg})$, and diastolic BP $(80.4 \pm 6.9 \mathrm{~mm} \mathrm{Hg})$. Diet logs indicated no significant differences for total calories, fat, carbohydrate, sodium, and protein intake among conditions. On average, male daily sodium intake was significantly higher than that of females $\left(2.7 \pm 0.3\right.$ vs. $\left.2.0 \pm 0.2 \mathrm{~g}, F_{[1,20]}=4.953, p=0.038\right)$. Physical activity level $24 \mathrm{~h}$ prior to data collection was not significantly different among conditions and remained low to sedentary. Due to contaminated samples, the final sample size for Posm, $\mathrm{P}\left[\mathrm{Na}^{+}\right]$, and $\mathrm{P}\left[\mathrm{K}^{+}\right]$varied among conditions (Control $=5, \mathrm{Npx}=3-4$, Heat $=4$, and NpxHeat $=3-4)$.

\section{Hydration}

Hydration measures for each experimental condition are presented in Table 1. No significant differences were found in hydration measures among conditions at any time point. All participants began trials euhydrated. Significant main effects for Posm $\left(F_{[2,26]}=5.165, p=0.013\right)$, Uosm $\left(F_{[2,66]}=17.257, p<0.001\right)$, and Usg $\left(F_{[2,80]}=10.081, p<0.001\right)$ occurred for time. Pairwise comparisons indicated that participants became more hydrated pre- to $3 \mathrm{~h}$ post-exercise (Table 1 ). All conditions maintained an average $\mathrm{BM}$ loss $<1 \%$.

Though not significantly different, mean Uvol was lower in the naproxen trials versus the placebo trial and during heat trials versus ambient trials (Table 2). There was no significant difference in Uvol between genders. Neither Fvol during exercise nor total Fvol was significantly different among conditions. Participants tended to consume more fluid during exercise in hot conditions versus ambient conditions (Table 2). Mean Fvol during exercise and overall was higher in both naproxen trials compared with placebo trials. This trend continued when corrected for BM. Participants' mean Fvol during 90 minutes of cycling $(1.5 \pm 0.7 \mathrm{~L})$ was significantly higher than their sweat rate $(0.9 \pm 0.3 \mathrm{~L} / \mathrm{h}$, $\left.t_{(10)}=-8.369, p=0.013\right)$. Compared with females, males consumed significantly greater Fvol during exercise $\left(1.1 \pm 0.5\right.$ and $1.7 \pm 0.7 \mathrm{~L}$, respectively, $\left.F_{[1,42]}=10.996, p=0.002\right)$ and total Fvol was also higher $\left(1.7 \pm 0.7\right.$ and $2.5 \pm 1.3$, respectively, $\left.F_{[1,41]}=6.854, p=0.012\right)$. During the NpxHeat trial, males consumed significantly more Fvol during exercise than females $\left(2.0 \pm 0.6\right.$ vs. $1.3 \pm 0.5 \mathrm{~L}, F_{[1,9]}=5.337$, $p=0.046)$. When corrected for BM, Fvol in males overall and during the NpxHeat trial was not 
Table 1. Hydration measures for the experimental conditions $(M \pm \mathrm{SD})$ before exercise (pre), immediately following exercise (post), $3 \mathrm{~h}$ after exercise ( $3 \mathrm{~h}$ post), and between one measurement time and another.

\begin{tabular}{|c|c|c|c|c|c|}
\hline & Aggregate & Control & Npx & Heat & NpxHeat \\
\hline \multicolumn{6}{|c|}{ Plasma osmolality (mOsm/L) } \\
\hline Pre & $286.6 \pm 6.4^{a}$ & $285.8 \pm 5.3$ & $288.8 \pm 4.8$ & $285.5 \pm 10.1$ & $287.8 \pm 6.6$ \\
\hline Post & $284.8 \pm 6.7$ & $288.2 \pm 6.6$ & $281.9 \pm 3.9$ & $285.9 \pm 10.6$ & $283.8 \pm 4.9$ \\
\hline $3 \mathrm{~h}$ post & $282.4 \pm 5.9$ & $286.4 \pm 3.3$ & $277.4 \pm 6.6$ & $283.6 \pm 6.9$ & $281.1 \pm 4.9$ \\
\hline \multicolumn{6}{|c|}{ Urine osmolality $(\mathrm{mOsm} / \mathrm{L})^{b}$} \\
\hline Pre & $399.5 \pm 172.1^{c}$ & $441.6 \pm 187.5$ & $431.8 \pm 193.5$ & $341.4 \pm 131.7$ & $389.9 \pm 183.4$ \\
\hline Post & $277.3 \pm 236.0$ & $246.8 \pm 223.7$ & $244.2 \pm 184.1$ & $233.1 \pm 213.9$ & $390.2 \pm 310.0$ \\
\hline $3 \mathrm{~h}$ post & $176.2 \pm 132.9$ & $192.4 \pm 107.8$ & $185.6 \pm 130.4$ & $201.3 \pm 173.0$ & $122.7 \pm 113.3$ \\
\hline \multicolumn{6}{|c|}{ Urine specific gravity } \\
\hline Pre & $1.012 \pm 0.005$ & $1.013 \pm 0.005$ & $1.014 \pm 0.006$ & $1.011 \pm 0.004$ & $1.012 \pm 0.004$ \\
\hline Post & $1.011 \pm 0.008$ & $1.010 \pm 0.007$ & $1.010 \pm 0.008$ & $1.010 \pm 0.008$ & $1.013 \pm 0.009$ \\
\hline $3 \mathrm{~h}$ post & $1.007 \pm 0.006^{d}$ & $1.007 \pm 0.005$ & $1.007 \pm 0.006$ & $1.007 \pm 0.006$ & $1.007 \pm 0.007$ \\
\hline \multicolumn{6}{|c|}{ Body mass change $(\%)$} \\
\hline Pre-post & $-0.1 \pm 0.8$ & $0.1 \pm 0.8$ & $-0.3 \pm 1.1$ & $0.0 \pm 0.6$ & $-0.1 \pm 0.8$ \\
\hline Pre-3 h post & $-0.4 \pm 0.9$ & $-0.6 \pm 0.4$ & $-0.1 \pm 0.6$ & $-0.5 \pm 0.6$ & $-0.5 \pm 0.9$ \\
\hline Post-3 h post & $-0.3 \pm 0.7$ & $-0.5 \pm 0.8$ & $0.0 \pm 1.3$ & $-0.4 \pm 0.5$ & $-0.0 \pm 0.6$ \\
\hline
\end{tabular}

Note: Npx, naproxen.

${ }^{a}$ Significantly higher than $3 \mathrm{~h}$ post- $(p=0.01)$ exercise.

${ }^{b}$ Significant main effect across time for all conditions $(p<0.001)$.

${ }^{c}$ Significantly higher than immediately post- $(p=0.003)$ and 3 h post- $(p<0.001)$ exercise.

${ }^{d}$ Significantly lower than pre- $(p<0.001)$ and immediately post- $(p=0.03)$ exercise.

Table 2. Fluid and urine volume for the experimental conditions $(M \pm \mathrm{SD})$.

\begin{tabular}{|c|c|c|c|c|c|}
\hline & Aggregate & Control & Npx & Heat & NpxHeat \\
\hline \multicolumn{6}{|c|}{ Urine volume $(\mathrm{mL})$} \\
\hline Total & $1012.9 \pm 519.1$ & $1157.3 \pm 681.4$ & $1075.3 \pm 572.3$ & $982.6 \pm 508.6$ & $836.6 \pm 214.2$ \\
\hline \multicolumn{6}{|c|}{ Fluid volume (L) } \\
\hline Exercise & $1.5 \pm 0.7$ & $1.1 \pm 0.4$ & $1.4 \pm 0.9$ & $1.6 \pm 0.6$ & $1.7 \pm 0.7$ \\
\hline Total & $2.1 \pm 1.1$ & $1.7 \pm 0.6$ & $2.1 \pm 1.5$ & $2.2 \pm 1.0$ & $2.5 \pm 1.3$ \\
\hline \multicolumn{6}{|c|}{ Fluid volume (mL/kg) } \\
\hline Exercise & $17.8 \pm 6.3$ & $14.2 \pm 4.2$ & $16.8 \pm 8.3$ & $19.6 \pm 4.9$ & $20.4 \pm 5.8$ \\
\hline Total & $25.9 \pm 10.0$ & $21.5 \pm 6.6$ & $25.5 \pm 13.4$ & $27.3 \pm 8.1$ & $29.5 \pm 10.6$ \\
\hline
\end{tabular}

Note: Npx, naproxen. No significant differences among the experimental conditions. 
significantly different than in females. During the Npx trial, overall Fvol approached significance (males $=1.9 \pm 1.0 \mathrm{~L}$ and females $\left.=0.9 \pm 0.5 \mathrm{~L}, F_{[1,9]}=4.859, p=0.055\right)$ and was different between genders during exercise with $\mathrm{BM}$ corrections (males $=20.7 \pm 8.8$, females $=12.1 \pm 5.0 \mathrm{~mL} / \mathrm{kg}$, $p=0.087)$.

\section{Electrolytes}

Table 3 presents plasma electrolytes by experimental condition. Mean aggregate $\mathrm{P}\left[\mathrm{Na}^{+}\right]$ was $<135 \mathrm{mmol} / \mathrm{L}$ pre-, immediately post-, and $3 \mathrm{~h}$ post-exercise. No statistically significant differences were found for $\mathrm{P}\left[\mathrm{Na}^{+}\right]$among conditions at any time. Mean $\mathrm{P}\left[\mathrm{Na}^{+}\right]$was highest during the NpxHeat trials. A significant main effect for $\mathrm{P}\left[\mathrm{K}^{+}\right]$was found over time $\left(F_{[2,26]}=4.287\right.$, $p=0.025)$, increasing pre- to post-exercise and then decreasing after $3 \mathrm{~h}$. No statistically significant differences among trials were observed, but both naproxen groups averaged higher $\mathrm{P}\left[\mathrm{K}^{+}\right]$ than placebo groups immediately post-exercise.

\section{Cardiovascular}

A significant main effect for HR occurred over time (mean pre $=66.8 \pm 14.3 \mathrm{bpm}$, immediately post $=177.4 \pm 16.2 \mathrm{bpm}$, and $3 \mathrm{~h}$ post $\left.=65.5 \pm 11.8 \mathrm{bpm}, F_{[1.7,67.4]}=1171.1, p<0.001\right)$. No differences among conditions or between genders were observed. A significant main effect occurred over time for systolic BP (mean pre $=118.3 \pm 1.6 \mathrm{~mm} \mathrm{Hg}$, immediately post $=137.4 \pm 2.8 \mathrm{~mm} \mathrm{Hg}$, and $\left.3 \mathrm{~h}=116.3 \pm 1.3 \mathrm{~mm} \mathrm{Hg}, F_{[1.6,63.2]}=58.989, p<0.001\right)$ and diastolic BP (mean pre $=73.2 \pm$ $10.5 \mathrm{~mm} \mathrm{Hg}$, immediately post $=71.7 \pm 10.1 \mathrm{~mm} \mathrm{Hg}$, and $3 \mathrm{~h}=76.9 \pm 7.8 \mathrm{~mm} \mathrm{Hg}, F_{[1.7,65.8]}=$ $5.602, p=0.008)$. No significant differences among conditions were observed. A significant main effect for systolic BP between genders was revealed $\left(F_{[1.7,70.5]}=6.241, p=0.005\right)$. Post hoc analysis showed that $\mathrm{BP}$ in males was significantly higher than in females at all time points (pre $=122.0 \pm 2.0$ vs. $113.8 \pm 2.2 \mathrm{~mm} \mathrm{Hg}, F_{[1,42]}=6.281, p=0.016$; immediately post $=146.3 \pm 3.1$ vs. $126.2 \pm 3.5 \mathrm{~mm}$ $\mathrm{Hg}, F_{[1,41]}=18.897, p<0.001$; and $3 \mathrm{~h}$ post $=119.7 \pm 1.5$ vs. $111.9 \pm 1.7 \mathrm{~mm} \mathrm{Hg}, F_{[1,42]}=13.11$, $p=0.001$; respectively). Male systolic BP was significantly higher than females immediately post-exercise in the $\mathrm{Npx}\left(150.8 \pm 19.6\right.$ vs. $\left.124.4 \pm 5.2 \mathrm{~mm} \mathrm{Hg}, F_{[1,9]}=8.457, p=0.017\right)$ and NpxHeat trials $\left(144.2 \pm 16.3\right.$ vs. $\left.123.5 \pm 5.5 \mathrm{~mm} \mathrm{Hg}, F_{[1,8]}=5.808, p=0.043\right)$.

Table 3. Plasma electrolytes for the experimental conditions $(M \pm \mathrm{SD})$ before exercise (Pre), immediately following exercise (Post), and $3 \mathrm{~h}$ after exercise ( $3 \mathrm{~h}$ post).

\begin{tabular}{|c|c|c|c|c|c|}
\hline & Aggregate & Control & Npx & Heat & NpxHeat \\
\hline \multicolumn{6}{|c|}{$\mathrm{P}\left[\mathrm{Na}^{+}\right](\mathrm{mmol} / \mathrm{L})$} \\
\hline Pre & $134.3 \pm 4.6$ & $133.5 \pm 5.7$ & $133.2 \pm 4.5$ & $133.5 \pm 3.2$ & $135.0 \pm 2.9$ \\
\hline Post & $133.5 \pm 3.2$ & $133.3 \pm 2.9$ & $131.5 \pm 4.8$ & $132.2 \pm 5.6$ & $134.8 \pm 3.9$ \\
\hline $3 \mathrm{~h}$ post & $134.3 \pm 3.1$ & $133.1 \pm 1.9$ & $133.5 \pm 0.3$ & $132.9 \pm 4.7$ & $135.3 \pm 2.7$ \\
\hline \multicolumn{6}{|c|}{$\mathbf{P}\left[\mathrm{K}^{+}\right](\mathbf{m m o l} / \mathrm{L})^{a}$} \\
\hline Pre & $3.9 \pm 0.4$ & $3.9 \pm 0.6$ & $4.1 \pm 0.4$ & $3.9 \pm 0.2$ & $3.6 \pm 0.1$ \\
\hline Post & $4.2 \pm 0.4$ & $4.2 \pm 0.7$ & $4.4 \pm 0.3$ & $3.9 \pm 0.1$ & $4.2 \pm 0.2$ \\
\hline $3 \mathrm{~h}$ post & $3.9 \pm 0.2$ & $3.8 \pm 0.3$ & $3.8 \pm 0.0$ & $4.0 \pm 0.3$ & $3.9 \pm 0.3$ \\
\hline
\end{tabular}

Note: $\mathrm{P}\left[\mathrm{K}^{+}\right]$, plasma potassium concentration; $\mathrm{P}\left[\mathrm{Na}^{+}\right]$, plasma sodium concentration; $\mathrm{Npx}$, naproxen.

${ }^{a}$ Significant main effect across time $(p=0.02)$. 


\section{Discussion}

We sought to determine whether naproxen adversely affected hydration and electrolyte balance during moderate-intensity endurance cycling in either hot or ambient conditions. Based on previous research (Cheng and Harris 2005), we hypothesized that NSAIDs would promote fluid retention through decreased Uvol and renal vasoconstriction, subsequently diluting plasma electrolytes, increasing hydration status, and increasing BP. Our results did not support our hypotheses, finding that an acute (24-h) naproxen dose did not significantly affect plasma electrolytes, plasma and urine hydration indices, or cardiovascular strain compared with placebos. We also report that responses did not differ between either hot or ambient environmental conditions.

\section{Fluid and electrolyte balance}

Naproxen is highly effective at inhibiting renal PGs, resulting in decreased renal blood flow (Garella and Matarese 1984). Specific renal consequences include decreased glomerular filtration rate, increased vasopressin, increased sodium retention, and decreased Uvol. Naproxen also suppresses the renin-angiotensin-aldosterone system, which is responsible for maintaining BP, plasma volume, and electrolyte balance (Cheng and Harris 2005). Independently or concurrently, naproxen's effects cause water retention and vasoconstriction (Cheng and Harris 2005). In extreme cases, cell necrosis, interstitial nephritis, and renal failure may develop (Garella and Matarese 1984). Milder consequences, although they can also become severe, include electrolyte imbalance, edema, and hypertension (Cheng and Harris 2005; Rosner and Kirven 2007).

Decreasing Uvol and increasing water retention is what makes NSAIDs a suspected contributor to hyponatremia. Despite this information, few studies have examined this relationship, with most being observational studies with conflicting results (Davis et al. 2001; Hsieh et al. 2002; Almond et al. 2005; Wharam et al. 2006). Ultra-distance triathletes using NSAIDs had significantly lower post-race $\mathrm{P}\left[\mathrm{Na}^{+}\right]$compared with non-NSAID users (Wharam et al. 2006). The six participants who experienced hyponatremia all reported using NSAIDs (Wharam et al. 2006). Of 15 marathon runners hospitalized with severe hyponatremia, 28.6\% used NSAIDs (Davis et al. 2001). In contrast, other observational studies have found no relationship between NSAIDs and hyponatremia (Hsieh et al. 2002; Almond et al. 2005; Scotney and Reid 2015). One inherent issue with existing literature is not knowing the NSAID type or dosage. All NSAIDs inhibit PGs, but their COX selectivity and chemical make-up, along with an individual person's unique response, means that each NSAID exerts its effects differently.

Another contributing factor for developing hyponatremia is an individual consuming hypotonic fluid in excess of fluid lost through sweat and respiration (Rosner and Kirven 2007). If combined with other factors that dilute $\mathrm{P}\left[\mathrm{Na}^{+}\right]$, the individual can be at greater risk for hyponatremia. Current recommendations advise matching individual sweat losses rather than drinking in excess or following generalized protocols (Casa et al. 2000; Sawka et al. 2007). Calculating sweat rate is relatively simple. However, lack of knowledge, ability, or resources may prohibit a person from calculating their sweat rate. Instead, the person may guestimate their fluid intake during endurance activity or determine it by trial and error. We chose to provide a standardized water protocol, rather than match sweat rates, and participants were allowed to consume more if desired. In part, using a standardized intake mimicked what an individual who does not know their personal water needs may do. Consuming water volumes exceeding sweat loss during 90 min of cycling did not significantly deplete $\mathrm{P}\left[\mathrm{Na}^{+}\right]$, and there was no effect from naproxen. The lack of $\mathrm{P}\left[\mathrm{Na}^{+}\right]$depletion is likely due to the short exercise duration. Exertional hyponatremia is typically associated with endurance exercise lasting longer than $3 \mathrm{~h}$ (Noakes et al. 2005; Rosner and Kirven 2007), but has also occurred in football (Creamer and

Hagedorn 2008; Blevins and Apel 2014; Stevens 2014). Our cycling session likely did not allow enough 
time to significantly deplete $\mathrm{P}\left[\mathrm{Na}^{+}\right]$. During the 3-h rest, when participants were allowed to drink water ad libitum they maintained euhydration and plasma electrolyte balance.

One interesting note is our mean $\mathrm{P}\left[\mathrm{Na}^{+}\right]$was $<135 \mathrm{mmol} / \mathrm{L}$ in all conditions and time points except pre- and $3 \mathrm{~h}$ post-exercise in the NpxHeat trials. Plasma sodium $<135 \mathrm{mmol} / \mathrm{L}$ without signs or symptoms is considered biochemical or asymptomatic hyponatremia (Noakes et al. 2005). Many individuals do not experience symptoms until $\mathrm{P}\left[\mathrm{Na}^{+}\right]$decreases below $130 \mathrm{mmol} / \mathrm{L}$, and severe hyponatremia typically occurs below $120 \mathrm{mmol} / \mathrm{L}$ (Sawka et al. 2007). Our participants maintained normal diets, consuming an average $2401.1 \pm 759.2 \mathrm{mg}$ of sodium daily. The low $\mathrm{P}\left[\mathrm{Na}^{+}\right]$can be explained by participants being instructed to arrive at the laboratory hydrated and by maintaining hydration during the exercise protocol. Pre-exercise Usg, Uosm, and Posm indicated that our participants were slightly hyperhydrated and became extremely hyperhydrated (Armstrong et al. 2010) by $3 \mathrm{~h}$ post-exercise.

It was also interesting that participants in the NpxHeat trials averaged more fluid consumed and had the lowest Uvol. The trend for naproxen trial participants to produce less urine while consuming more fluid suggests that participants were retaining water. We did not see significant decreases in $\mathrm{P}\left[\mathrm{Na}^{+}\right]$, Posm, or Usg pre- to post-exercise. The low sample size for $\mathrm{P}\left[\mathrm{Na}^{+}\right]$and Posm makes it difficult to definitively assess whether fluid was retained during naproxen trials. However, we presume a portion of water remained in the stomach. Intense exercise, such as with our cycling protocol, slows gastric emptying, which prevents fluid from being absorbed and (or) used to maintain physiological processes (Robinson et al. 1995).

In principle, increased Fvol and decreased Uvol should lead to BM gains. No significant differences in BM were found among our experimental conditions. Both the Npx and NpxHeat trial participants averaged pre- to post-exercise BM losses, whereas both Control and Heat trial participants showed a slight increase in BM or no change (Table 1). From immediately post- to $3 \mathrm{~h}$ post-exercise, this trend flipped with Control and Heat trial participants showing more weight loss than Npx and NpxHeat trial participants. Once exercise ceased, it is possible that gastrointestinal and renal function returned to normal, promoting gastric emptying and water excretion as necessary to maintain fluidelectrolyte balance. Support for this explanation is found with the slightly higher Uvol in Control and Heat treatments. In contrast, the continuation of naproxen's effects throughout the rest period would explain the slight weight gain and lower average Posm $3 \mathrm{~h}$ post-exercise. This is important to note considering reported cases of hyponatremia developing hours after activity (Creamer and Hagedorn 2008; Stevens 2014). Sustained water retention combined with continued hypotonic fluid consumption would place the person at even greater risk for diluted $\mathrm{P}\left[\mathrm{Na}^{+}\right]$.

Our participants remained within normal $\mathrm{P}\left[\mathrm{K}^{+}\right]$levels $(<5 \mathrm{mmol} / \mathrm{L})$ (Clausen 2010). Potassium is tightly regulated by the kidneys because elevated plasma levels may cause cardiac arrhythmias and death (Clausen 2010). Even during exercise, when plasma levels can quickly spike due to working muscles releasing or failing to re-uptake potassium (Clausen 2010), the kidneys work efficiently to clear potassium. However, potassium will stay elevated if renal blood flow is decreased (Franscesconi et al. 1997). NSAIDs, known to decrease renal blood flow (Garella and Matarese 1984), can induce hyperkalemia (Lafrance and Miller 2012), but little scientific information is available regarding this association during exercise. The increase in our overall $\mathrm{P}\left[\mathrm{K}^{+}\right]$mean values are similar to those reported in existing literature (Wharam et al. 2006; Dumke et al. 2007; McKenney et al. 2015). Although not significantly different among conditions, the higher means for naproxen compared with placebos suggest that there could be some effect from the NSAID. Among triathletes, NSAIDs resulted in significantly higher post-race $\mathrm{P}\left[\mathrm{K}^{+}\right]$compared with those not using NSAIDs (Wharam et al. 2006). In contrast, over-the-counter ibuprofen did not significantly affect $\mathrm{P}\left[\mathrm{K}^{+}\right]$in ultra-distance runners (Dumke et al. 2007). The type of NSAID was not reported by Wharam et al. (2006); therefore, it is not possible to make comparisons among specific NSAIDs. However, research 
has established that, compared with no NSAID exposure, the odds for developing hyperkalemia are higher with the use of naproxen than with ibuprofen (Lafrance and Miller 2012). Using a higher naproxen dose for a longer duration may induce significant $\mathrm{P}\left[\mathrm{K}^{+}\right]$changes during exercise.

\section{Cardiovascular}

Our results indicate 3-220 mg naproxen doses over $24 \mathrm{~h}$ did not significantly affect HR or BP. Cardiovascular responses often occur concurrently with renal responses, because altering fluidelectrolyte balance can control plasma volume and BP. Disregarding renal effects, naproxen is less effective at directly altering the cardiovascular system because naproxen is more COX-2 selective (Botting 2006). Further, naproxen reversibly inhibits platelet aggregation. In comparison, aspirin is more selective toward COX-1 and irreversibly inhibits platelet aggregation, making aspirin highly effective at reducing cardiovascular events (i.e., stroke, heart attack) (Patrono et al. 2005). Lack of cardiovascular effects could be attributed to naproxen itself or to our methodology. By maintaining hydration, we limited cardiovascular changes that would otherwise be seen with hypohydration. We also limited our participants to healthy, young, moderately trained individuals. The same cardiovascular results may not be seen in different populations (e.g., older, poor physical conditioning, cardiovascular or renal disease).

\section{Gender}

The current literature indicates that females are at higher risk for hyponatremia because of smaller BM, excessive water intake, and longer race times compared with males (Rosner and Kirven 2007). Though these factors play a role, hormonal differences between genders are also an important consideration. Resting plasma vasopressin varies in females depending on menstrual phase. Vasopressin is significantly lower than males during the early follicular phase, but not during the luteal phase (Wenner and Stachenfeld 2012). These variances may help explain why we found differences in Fvol between genders. Males consumed significantly more fluid than females both during exercise and overall. Interestingly, males consumed significantly more fluid during exercise in the NpxHeat trials, and approached significance in the Npx trials, compared with females. Gender differences did not occur during the Control and Heat trials, and we found no difference in females' Fvol among conditions. Considering the change in vasopressin during menstrual phases, which we did not account for, the lack of significant findings among females could be partially explained by hormones.

Thirst is listed as a potential side effect for several NSAIDs, but data are limited for the effect of NSAIDs on thirst or Fvol during exercise. Among NSAID and non-NSAID users completing an $82 \mathrm{~km}$ mountain run, NSAID users consumed significantly more fluid than non-users (Scotney and Reid 2015). Our results support that naproxen use increases fluid intake in males. We used naproxen sodium, which is bioequivalent to naproxen except for the rate of absorption. The salt of a given NSAID is commonly used because it allows the drug to dissolve faster and exert effects earlier. A $220 \mathrm{mg}$ naproxen sodium dose contains approximately $20 \mathrm{mg}$ of sodium. Not all salts exert the same effects and $20 \mathrm{mg}$ is relatively low, but $46 \mathrm{mg} / \mathrm{L}$ of sodium chloride added to a flavored beverage significantly increased fluid intake (Wemple et al. 1997). A possible explanation for increased Fvol in our male participants is that naproxen sodium stimulated thirst. Another mechanism for thirst stimulation is an increase in vasopressin from NSAIDs (Cheng and Harris 2005). Vasopressin is typically upregulated during dehydration to restore plasma volume, and vasopressin increase is positively and linearly associated with thirst (Wenner and Stachenfeld 2012). Despite our participants being euhydrated, if naproxen stimulated vasopressin it could have increased their fluid intake.

We identified no differences between or within genders for HR. BP is generally higher for males than females (Christou et al. 2005), which is attributed to physical differences, hormones, and vascular responsiveness (Wenner and Stachenfeld 2012). Male baseline BP was slightly higher than females, 
but not significantly. Naproxen significantly elevated post-exercise male systolic BP compared with females. This did not occur during Control and Heat trials. The increased Fvol with naproxen in males could partially explain the higher systolic BP. Ostensibly, males would have a higher plasma volume, which would increase BP.

\section{Limitations and future research}

Measuring aldosterone and vasopressin would provide better insight into how naproxen influences fluid and electrolyte balance. Measuring plasma naproxen concentration would have been useful to determine the drug's concentration $3 \mathrm{~h}$ post-exercise. Naproxen has a long half-life compared with other NSAIDs. Therefore, naproxen is expected to continue exerting effects during the 3 -h rest, whereas other NSAIDs would not. This is important when considering post-activity effects. Our 90-min cycling was likely too short to elicit significant $\mathrm{P}\left[\mathrm{Na}^{+}\right]$changes, considering most hyponatremia research is in marathon and ultra-distance events. Our sample size for plasma electrolytes and Posm was low, preventing us from achieving statistical significance among conditions for Posm, $\mathrm{P}\left[\mathrm{Na}^{+}\right]$, and $\mathrm{P}\left[\mathrm{K}^{+}\right]$. This also limited our ability to extensively examine cardiovascular effects. Finally, we did not control for menstrual phase.

Future research is warranted to determine the relationship between NSAID use and hydrationelectrolyte balance during exercise. Because each NSAID is unique, studies should evaluate different types, dosages (e.g., over-the-counter, prescription), and length of use (e.g., 3 d, 2 weeks). Evaluating longer endurance exercise periods to deplete plasma electrolytes is an important consideration. Longer exercise bouts, particularly marathon and ultra-distance events, is also important due to the high NSAID use among these athletes. At the same time, examining shorter exercise periods is relevant considering the potential for electrolyte depletion due to other risk factors such as inadequate nutrition, excessive hypotonic fluid intake, and (or) excessive sweat sodium loss. Using different hydration regimens (e.g., metered versus ad libitum), fluid types (e.g., carbohydrate-electrolyte beverages), and hydration statuses (e.g., severe hypohydration) is also merited. Considering the trend of increased Fvol with naproxen use, evaluating vasopressin and thirst would be interesting. Along these lines, examining the salts of different NSAID types would provide meaningful information. Finally, controlled studies evaluating markers of renal function and gender differences, including differences during menstrual phases, are merited.

\section{Conclusion}

A 24-h naproxen dose did not adversely affect hydration, electrolyte, or cardiovascular measures among healthy, hydrated males and females cycling for $90 \mathrm{~min}$ in either hot or ambient conditions. Although our participants maintained electrolyte balance, their $\mathrm{P}\left[\mathrm{Na}^{+}\right]$was lower than $135 \mathrm{mmol} / \mathrm{L}$. Finding higher post-exercise systolic BP with naproxen use in males versus females warrants consideration for potential adverse events during exercise in males with pre-existing hypertension. Further, we found that naproxen use promoted greater fluid intake in males in ambient and hot environments. Beginning exercise slightly hyperhydrated and consuming water in excess of the sweat rate during our 90 min exercise time began to show a trend for naproxen use to induce water retention through decreased Uvol. Together, these results suggest that medical personnel should consider an individual's risk factors (i.e., gender, fluid intake, existing renal or cardiovascular conditions) when recommending naproxen use during exercise.

\section{Acknowledgements}

Portions of the study were supported by grant funding received from the National Athletic Trainers' Association Research and Education Foundation and the American College of Sports Medicine 
Foundation. The authors would like to thank the following individuals for their invaluable assistance throughout this study: Dr. Brian Keisler, Dr. Reilly Enos, Dr. Angela Murphy, Kei Lam, and Hawthorne Pharmacy. Thank you to Mrs. Amy Hand and Ms. Elena Keretses for assisting with data collection.

\section{Author contributions}

DME, TMT-M, JMD, SCLC, JLD, and CCE conceived and designed the study. DME, TMT-M, CEP, CCE, JVS, and JDB performed the experiments/collected the data. DME, TMT-M, JMD, SCLC, and JLD analyzed and interpreted the data. DME, TMT-M, JMD, and SCLC contributed resources. DME, TMT-M, JMD, SCLC, JLD, CEP, CCE, JVS, and JDB drafted or revised the manuscript.

\section{Competing interests}

The authors have declared that no competing interests exist.

\section{Data accessibility statement}

All relevant data are within the paper.

\section{References}

Almond CSD, Shin AY, Fortescue EB, Mannix RC, Wypij D, Binstadt BA, et al. 2005. Hyponatremia among runners in the Boston Marathon. The New England Journal of Medicine, 352(15): 1550-1556. PMID: 15829535 DOI: 10.1056/NEJMoa043901

Armstrong LE, Pumerantz AC, Fiala KA, Roti MW, Kavouras SA, Casa DJ, et al. 2010. Human hydration indices: acute and longitudinal reference values. International Journal of Sport Nutrition and Exercise Metabolism, 20(2): 145-153. PMID: 20479488 DOI: 10.1123/ijsnem.20.2.145

Blevins R, and Apel T. 2014. Doctor: Wilbanks' death unpreventable, freak occurrence. The ClarionLedger [online]: Available from clarionledger.com/story/prepsreport/2014/08/25/walker-wilbankscause-of-death-related-to-over-hydration/14598215/.

Botting RM. 2006. Inhibitors of cyclooxygenases: mechanisms, selectivity and uses. Journal of Physiology and Pharmacology, 57: 113-124. PMID: 17218763

Casa DJ, Armstrong LE, Hillman SK, Montain SJ, Reiff RV, Rich BSE, et al. 2000. National Athletic Trainers' Association position statement: fluid replacement for athletes. Journal of Athletic Training, 35(2): 212-224. PMID: 16558633

Cheng HF, and Harris RC. 2005. Renal effects of non-steroidal anti-inflammatory drugs and selective cyclooxygenase-2 inhibitors. Current Pharmaceutical Design, 11(14): 1795-1804. PMID: 15892676 DOI: $10.2174 / 1381612053764922$

Christou DD, Jones PP, Jordan J, Diedrich A, Robertson D, and Seals DR. 2005. Women have lower tonic autonomic support of arterial blood pressure and less effective baroreflex buffering than men. Circulation, 111(4): 494-498. PMID: 15687139 DOI: 10.1161/01. CIR.0000153864.24034.A6

Clausen T. 2010. Hormonal and pharmacological modification of plasma potassium homeostasis. Fundamental \& Clinical Pharmacology, 24(5): 595-605. PMID: 20618871 DOI: 10.1111/ j.1472-8206.2010.00859.x 
Constanti A, Bartke A, and Khardori R. 1998. Basic endocrinology. Harwood Academic Publishers, Amsterdam, the Netherlands.

Creamer M, and Hagedorn E. 2008. Family in disbelief that drinking too much water killed football player. The Bakersfield Californian [online]: Available from bakersfield.com/archives/ family-in-disbelief-that-drinking-too-much-water-killed-football/article_bbbe0492-fc40-5fd8-baa9d220070428ff.html.

Davis DP, Videen JS, Marino A, Vilke GM, Dunford JV, Van Camp SP, et al. 2001. Exercise-associated hyponatremia in marathon runners: a two-year experience. The Journal of Emergency Medicine, 21(1): 47-57. PMID: 11399389 DOI: 10.1016/S0736-4679(01)00320-1

Dumke CL, Nieman DC, Oley K, and Lind RH. 2007. Ibuprofen does not affect serum electrolyte concentrations after an ultradistance run. British Journal of Sports Medicine, 41(8): 492-496. PMID: 17331976 DOI: 10.1136/bjsm.2006.033597

Faul F, Erdfelder E, Lang A-G, and Buchner A. 2007. G*Power 3: a flexible statistical power analysis program for the social, behavioral, and biomedical sciences. Behavior Research Methods, 39(2): 175-191. PMID: 17695343 DOI: 10.3758/BF03193146

Franscesconi RP, Willis JS, Gaffin SL, and Hubbard RW. 1997. On the trail of potassium in heat injury. Wilderness and Environmental Medicine, 8: 105-110. PMID: 11990141 DOI: 10.1580/ 1080-6032(1997)008[0105:OTTOPI]2.3.CO;2

Garella S, and Matarese RA. 1984. Renal effects of prostaglandins and clinical adverse effects of nonsteroidal anti-inflammatory agents. Medicine, 63(3): 165-181. PMID: 6371441 DOI: $10.1097 /$ 00005792-198405000-00003

Hsieh M, Roth R, Davis DL, Larrabee H, and Callaway CW. 2002. Hyponatremia in runners requiring on-site medical treatment at a single marathon. Medicine and Science in Sports and Exercise, 34(2): 185-189. PMID: 11828223 DOI: 10.1097/00005768-200202000-00001

Lafrance J-P, and Miller DR. 2012. Dispensed selective and nonselective nonsteroidal anti-inflammatory drugs and the risk of moderate to severe hyperkalemia: a nested case-control study. American Journal of Kidney Disease, 60(1): 82-89. PMID: 22503390 DOI: 10.1053/j.ajkd.2012.02.328

McKenney MA, Miller KC, Deal JE, Garden-Robinson JA, and Rhee YS. 2015. Plasma and electrolyte changes in exercising humans after ingestion of multiple boluses of pickle juice. Journal of Athletic Training, 50(2): 141-146. PMID: 25562454 DOI: 10.4085/1062-6050-50.2.07

Noakes TD, Sharwood K, Speedy D, Hew T, Reid S, Dugas J, et al. 2005. Three independent biological mechanisms cause exercise-associated hyponatremia: evidence from 2,135 weighed competitive athletic performances. Proceedings of the National Academy of Sciences of the USA, 102(51): 18550-18555. PMID: 16344476 DOI: 10.1073/pnas.0509096102

Patrono C, García Rodríguez LA, Landolfi R, and Baigent C. 2005. Low-dose aspirin for the prevention of atherothrombosis. The New England Journal of Medicine, 353: 2373-2383. PMID: 16319386 DOI: 10.1056/NEJMra052717

Peters HPF, Wiersma WC, Akkermans LM, Bol E, Kraaijenhagen RJ, Mosterd WL, et al. 2000. Gastrointestinal mucosal integrity after prolonged exercise with fluid supplementation. Medicine and Science in Sports and Exercise, 32(1): 134-142. PMID: 10647540 DOI: 10.1097/ 00005768-200001000-00020 
Robinson TA, Hawley JA, Palmer GS, Wilson GR, Gray DA, Noakes TD, et al. 1995. Water ingestion does not improve 1-h cycling performance in moderate ambient temperatures. European Journal of Applied Physiology and Occupational Physiology, 71: 153-160. PMID: 7588683 DOI: 10.1007/ BF00854973

Rosner MH, and Kirven J. 2007. Exercise-associated hyponatremia. Clinical Journal of the American Society of Nephrology, 2(1): 151-161. PMID: 17699400 DOI: 10.2215/CJN.02730806

Sawka MN, Burke LM, Eichner ER, Maughan RJ, Montain SJ, and Stachenfeld NS. 2007. American College of Sports Medicine position stand. Exercise and fluid replacement. Medicine and Science in Sports and Exercise, 39(2): 377-390. PMID: 17277604 DOI: 10.1249/mss.0b013e31802ca597

Scotney B, and Reid S. 2015. Body weight, serum sodium levels, and renal function in an ultradistance mountain run. Clinical Journal of Sport Medicine, 25: 341-346. PMID: 25010155 DOI: $10.1097 / J S M .0000000000000131$

Stevens A. 2014. Update: Douglas County football player has died. The Atlanta Journal-Constitution [online]: Available from ajc.com/news/update-douglas-county-football-player-has-died/ G73yyxV1Sxo4N1IMdcibQO/.

Wemple RD, Morocco TS, and Mack GW. 1997. Influence of sodium replacement on fluid ingestion following exercise-induced dehydration. International Journal of Sport Nutrition, 7: 104-116. PMID: 9189781 DOI: 10.1123/ijsn.7.2.104

Wenner MM, and Stachenfeld NS. 2012. Blood pressure and water regulation: understanding sex hormone effects within and between men and women. The Journal of Physiology, 590(23): 5949-5961. PMID: 23027816 DOI: 10.1113/jphysiol.2012.236752

Wharam PC, Speedy DB, Noakes TD, Thompson JM, Reid SA, and Holtzhausen LM. 2006. NSAID use increases the risk of developing hyponatremia during an Ironman triathlon. Medicine and Science in Sports and Exercise, 38(4): 618-622. PMID: 16679974 DOI: 10.1249/01.mss. 0000210209.40694.09 